advertisements, and a good match between websites, banner material and target population. This specific campaign design aids the recruitment of subjects for a preclinical Alzheimer's disease trial.

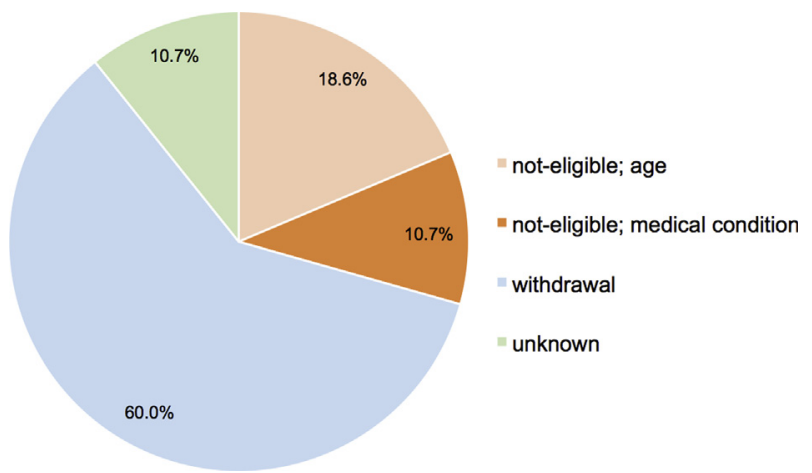

Figure 3. Main reasons for drop-out during the pre-screening process.

Table 1

Specifications of different reasons for drop-out during prescreening as shown in Figure 3.

\begin{tabular}{lc} 
Reason drop-out & Drop-outs $(\mathrm{n}=177)$ \\
\hline Not-eligible; age & 33 \\
Age $<60$ years & 18 \\
Age $>75$ years & 15 \\
Not-eligible; medical condition & 19 \\
Malignancy & 9 \\
Psychiatric disorder & 1 \\
Alzheimer's disease & 1 \\
MRI contra-indication & 2 \\
Other & 6 \\
Withdrawal & 106 \\
Travel distance & 24 \\
Reluctant to find out APOE genotype & 7 \\
Trial too demanding & 5 \\
Unwilling to take experimental & 3 \\
$\quad$ medication & \\
Unwilling to provide personal & 2 \\
$\quad$ information & \\
Concerns about privacy of personal data & 4 \\
Information in ICF unclear & 1 \\
Insufficient financial compensation & 4 \\
Unspecified & 56 \\
Unknown & 19
\end{tabular}

Abbreviations: $\mathrm{MRI}=$ magnetic resonance imaging; $\mathrm{APOE}=$ Apolipoprotein E; ICF = informed consent form.

\section{P3-022 THE ALZHEIMER'S PREVENTION INITIATIVE (API) PROGRAM: DESIGN AND DEVELOPMENT OF AN APOE COUNSELING AND DISCLOSURE PROGRAM}

Carolyn Langlois ${ }^{1}$, Jason Karlawish ${ }^{2}$, Angela R. Bradbury ${ }^{2}$, Elisabeth McCarty Wood ${ }^{2}$, J. Scott Roberts ${ }^{3}$, Scott Kim ${ }^{4}$, Linda PatrickMiller $^{5}$, Eric M. Reiman ${ }^{6}$, Pierre N. Tariot ${ }^{1}$, Jessica B. Langbaum ${ }^{6},{ }^{1}$ Banner Alzheimer's Institute, Phoenix, AZ, USA; ${ }^{2}$ University of Pennsylvania, Philadelphia, PA, USA, ${ }^{3}$ University of Michigan, Ann Arbor, MI, USA;
${ }^{4}$ National Institutes of Health, Bethesda, MD, USA; ${ }^{5}$ Independent consultant, Chicago, IL, USA; ${ }^{6}$ Arizona Alzheimer's Consortium, Phoenix, AZ, USA. Contacte-mail: carolyn.langlois@bannerhealth.com

Background: The Alzheimer's Prevention Initiative (API) is a collaborative funded by the NIH, philanthropy, and industry to conduct preclinical Alzheimer's disease (AD) trials in people who, based on age and genetics, are at elevated risk for developing AD symptoms. The API Generation Program is enrolling apolipoprotein E (APOE) $e 4$ carriers age 60-75. To support disclosure of APOE genotype, API established an interdisciplinary Genetic Testing and Disclosure Committee to guide development of the API Genetic Counseling and Disclosure Program (GCDP). Methods: The API GCDP is being implemented globally in a manner that accommodates varying local laws and regulations, different referral sources, and persons who are, or are not, already aware of their APOE genotype. A pre-disclosure educational video informs participants about AD, APOE, and considerations for learning their APOE results. To assist in clearly and safely communicating genetic information, a risk-disclosure handout, guided talking points, and genotype-specific summary sheets are used as part of the disclosure process. Participants are assessed with measures of genetic knowledge, psychological wellbeing, health behaviors, impact of disclosure, and satisfaction with disclosure at multiple time points following the disclosure session to examine the impact of learning APOE one's results. An ancillary sub-study in the US (CONNECT4 APOE) compares telephone vs videoconference delivery of APOE genetic counseling and disclosure, while an online sub-study (Cognition Across Time) will examine whether APOE disclosure impacts subjective and objective memory and thinking ability. Results: As of 30-Oct-2017, 209 participants have undergone APOE genetic disclosure as part of screening for the Generation Program, of whom 144 learned their APOE results for the first time. As of 8-Jan-2018, 141 participants have received remote disclosure as part of their participation in CONNECT4 APOE. Quantitative and qualitative feedback regarding the API GCDP is collected in an ongoing manner at the Sponsor, Genetic Counselor and participant level. Conclusions: The API GCDP is a key component of the API program, intended to facilitate clear and safe communication of genetic information for current and future API trials. Information collected will allow for ongoing refinement and improvement of the API GCDP as well as provide data relevant for future studies in genetically enriched populations.

\section{P3-023 BEYOND ANTIPSYCHOTICS: EXPLORING EFFICACY AND HARMS OF Z-DRUGS FOR SLEEP DISTURBANCE ON THE PROGRESSION OF KEY DEMENTIA OUTCOMES}

Chris Fox ${ }^{1}$, Antony Arthur ${ }^{1}$, Clive Ballard ${ }^{2}$, Robert J. Howard ${ }^{3}$, Ian D. Maidment ${ }^{4}$, Eduwin Pakpahan ${ }^{5}$, Kathryn Richardson ${ }^{1}$, Penelope Boyd ${ }^{1}$, Clare Aldus ${ }^{1}$, Yoon Loke ${ }^{1}$, Nick Steel ${ }^{1}$, George M. Savva ${ }^{1}$, ${ }^{1}$ University of East Anglia, Norwich, United Kingdom; ${ }^{2}$ University of Exeter Medical School, Exeter, United Kingdom; ${ }^{3}$ University College London, London, United Kingdom; ${ }^{4}$ Aston University, Birmingham, United Kingdom; ${ }^{5}$ Newcastle University, Newcastle, United Kingdom. Contact e-mail: chris.fox@uea.ac.uk

Background: Approximately $60 \%$ of people with dementia (PwD) experience sleep disturbance including insomnia and excessive daytime sleeping. Hypnotic Z-drugs (zolpidem, zopiclone and 